\title{
Influence of trigger type, tube voltage and heart rate on calcified plaque imaging in dual source cardiac computed tomography: phantom study
}

\author{
Tobias Penzkofer ${ }^{1,2,3^{*}}$, Eva Donandt ${ }^{1}$, Peter Isfort ${ }^{1,3}$, Thomas Allmendinger ${ }^{4}$, Christiane K Kuhl ${ }^{1}$,
} Andreas H Mahnken ${ }^{1,3,5}$ and Philipp Bruners ${ }^{1,3}$

\begin{abstract}
Background: To investigate the impact of high pitch cardiac $C T$ vs. retrospective ECG gated $C T$ on the quantification of calcified vessel stenoses, with assessment of the influence of tube voltage, reconstruction kernel and heart rate.

Methods: A 4D cardiac movement phantom equipped with three different plaque phantoms (12.5\%, 25\% and 50\% stenosis at different calcification levels), was scanned with a 128-row dual source CT scanner, applying different trigger types (gated vs. prospectively triggered high pitch), tube voltages (100-120 kV) and heart rates (50-90 beats per minute, bpm). Images were reconstructed using different standard (B26f, B46f, B70f) and iterative (I26f, I70f) convolution kernels. Absolute and relative plaque sizes were measured and statistically compared. Radiation dose associated with the different methods (gated vs. high pitch, 100 kV vs. 120 kV) were compared.

Results: Compared to the known diameters of the phantom plaques and vessels both CT-examination techniques overestimated the degrees of stenoses. Using the high pitch CT-protocol plaques appeared larger $(0.09 \pm 0.31 \mathrm{~mm}$, $2 \pm 8$ percent points, PP) in comparison to the ECG-gated (T-scans. Reducing tube voltage had a similar effect, resulting in higher grading of the same stenoses by $3 \pm 8 \mathrm{PP}$. In turn, sharper convolution kernels lead to a lower grading of stenoses (differences of up to 5\%). Pairwise comparison of B26f and I26f, B46f and B70f, and B70f and I70f showed differences of 0-1 \pm 6-8 PP of the plaque depiction. Motion artifacts were present only at $90 \mathrm{bpm}$ high pitch experiments. High-pitch protocols were associated with significantly lower radiation doses compared with the ECG-gated protocols (258.0 mGy vs. 2829.8 mGy CTDI $I_{\text {vol, }} \mathrm{p} \leq 0.0001$ ).

Conclusion: Prospectively triggered high-pitch cardiac CT led to an overestimation of plaque diameter and degree of stenoses in a coronary phantom. This overestimation is only slight and probably negligible in a clinical situation. Even at higher heart rates high pitch CT-scanning allowed reliable measurements of plaque and vessel diameters with only slight differences compared ECG-gated protocols, although motion artifacts were present at $90 \mathrm{bpm}$ using the high pitch protocols.
\end{abstract}

\section{Background}

Cardiac computed tomography (CT) is an established non-invasive method of assessing coronary artery morphology both in emergency and routine settings [1]. It is superior to magnetic resonance based methods of coronary angiography with respect to temporal and spatial

\footnotetext{
* Correspondence: tpenzkofer@ukaachen.de

'Department of Diagnostic and Interventional Radiology, Aachen University Hospital, RWTH Aachen University, Pauwelsstr. 30, 52074 Aachen, Germany ${ }^{2}$ Surgical Planning Laboratory, Department of Radiology, Brigham and Women's Hospital, 75 Francis Street, 02115 Boston, USA

Full list of author information is available at the end of the article
}

resolution. However, continuous motion of the heart makes cardiac cross-sectional imaging a challenging task [1,2]. Different methods have been developed to overcome this problem, many of them rely on the uniformity of the cardiac cycle by imaging different sections of the heart over several consecutive heart beats. To date the vast majority of current CT systems uses either a prospectively ECG-triggered or retrospectively ECG-gated scanning protocol for this purpose. Since all these methods are associated with ionizing radiation applied to the patient, the risks and benefits need to be carefully weighted [3].

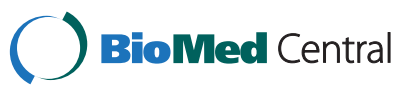

(c) 2014 Penzkofer et al.; licensee BioMed Central Ltd. This is an Open Access article distributed under the terms of the Creative Commons Attribution License (http://creativecommons.org/licenses/by/2.0), which permits unrestricted use, distribution, and reproduction in any medium, provided the original work is properly credited. The Creative Commons Public Domain Dedication waiver (http://creativecommons.org/publicdomain/zero/1.0/) applies to the data made available in this article, unless otherwise stated. 


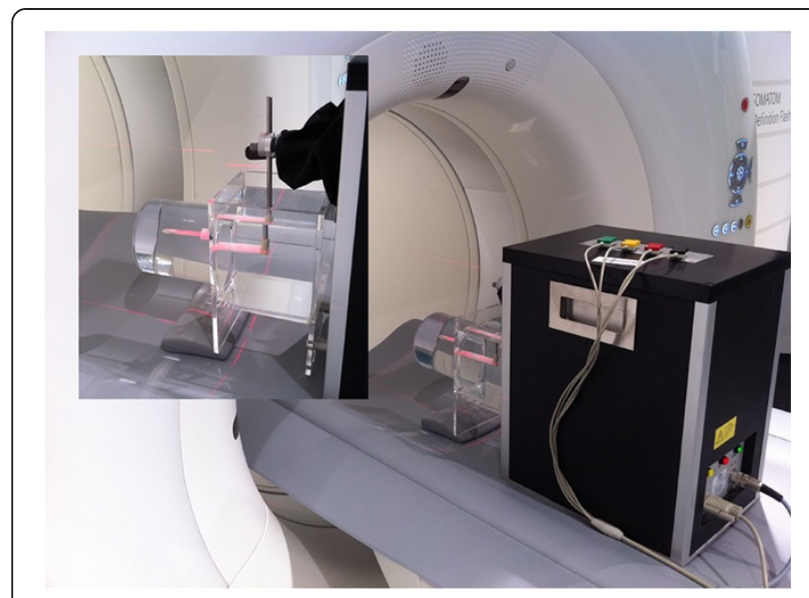

Figure 1 Cardiac movement phantom setting during the scans. (inlay) the coronary artery phantom (CAP) was placed in a water filled container. The cardiac phantom provides 4D motion with ECG-syncing over the scanners standard ECG-interface (ECGI).

Recent advances in CT technology led to the development of dual source based high pitch (pitch up to 3.4) scanning protocols with table speeds of up to $46 \mathrm{~cm} / \mathrm{sec}$ [4-6], or even higher [7,8]. These protocols promise to image the heart within one heartbeat, obviating the need for gating or multi-step triggering, i.e. acquisition techniques that are inherently associated with redundant scan ranges leading to increased radiation doses.

The goal of this study was to evaluate the quantification of calcified stenosis in high pitch cardiac CT-scans versus an ECG-gated scan protocol in a controlled phantom environment. Secondary goal was to evaluate the influence of tube current, convolution kernel and heart rate on the different acquisition protocols.

\section{Methods}

\section{Coronary movement phantom}

A 4D coronary movement simulator (Sim4Dcardiac, QRM GmbH, Möhrendorf, Germany, Figure 1) was used for the study performing coronary artery movement patterns with heart rates of 50,70 and 90 beats per minute (bpm). Three custom made dedicated coronary artery phantoms (QRM GmbH, Möhrendorf, Germany) each with defined calcified (high, medium and low calcification at 796,401 and $197 \mathrm{mgHA} / \mathrm{cm}^{3}$ at densities of $1.58,1.30$ and $1.16 \mathrm{~g} / \mathrm{cm}^{3}$ ) coronary stenoses of various degrees $(50 \%, 25 \%$ and $12.5 \%)$ were used in a simulated $4.0 \mathrm{~mm}$ vessel. The absolute stenosis sizes were $0.5 \mathrm{~mm}(12.5 \%)$,

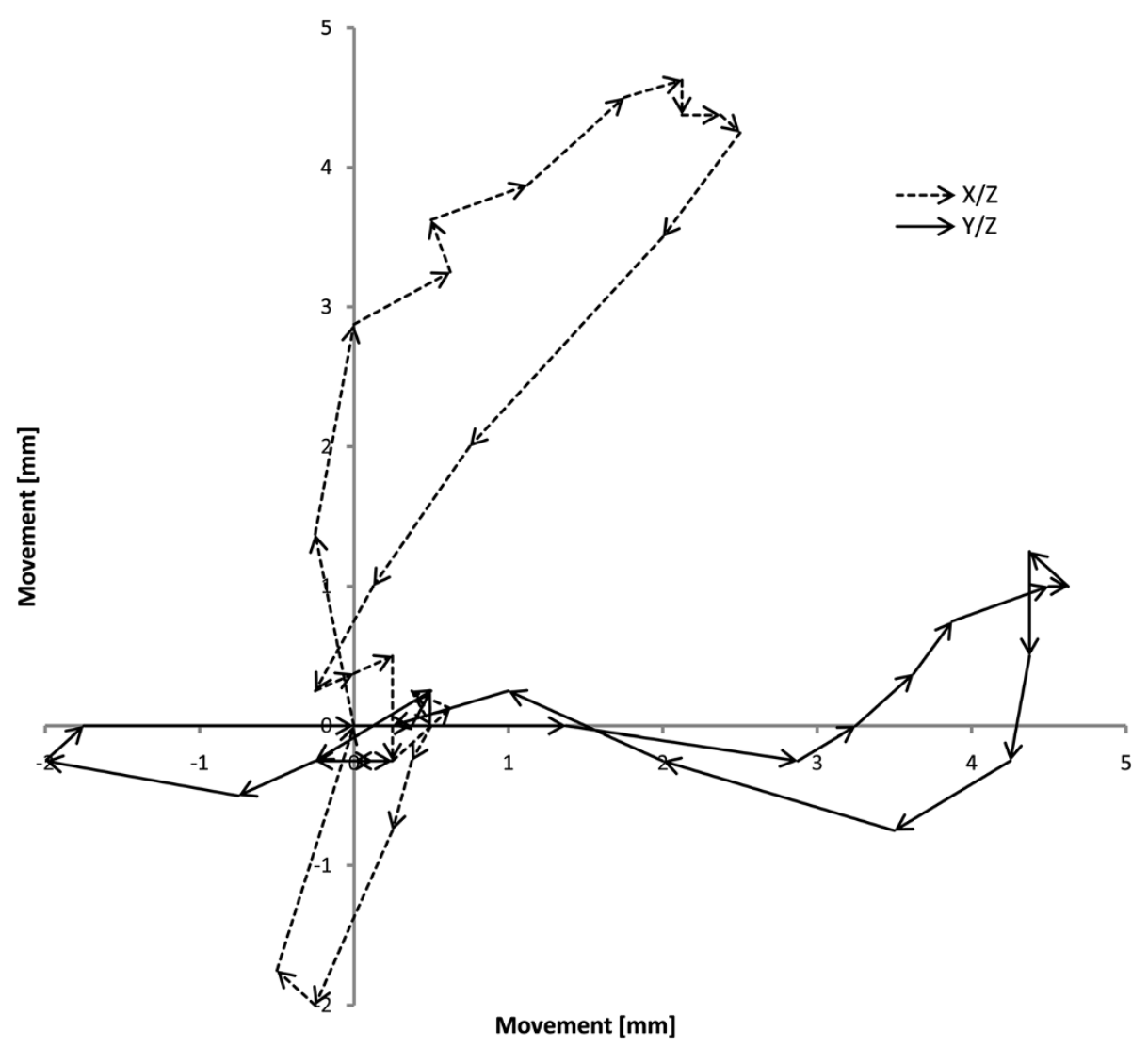

Figure 2 Cardiac movement as performed by the coronary movement simulator. The solid line represents the movement in the Y/Z plane, while the dashed line shows the path in the X/Z plane. Arrow length corresponds to movement speed. 


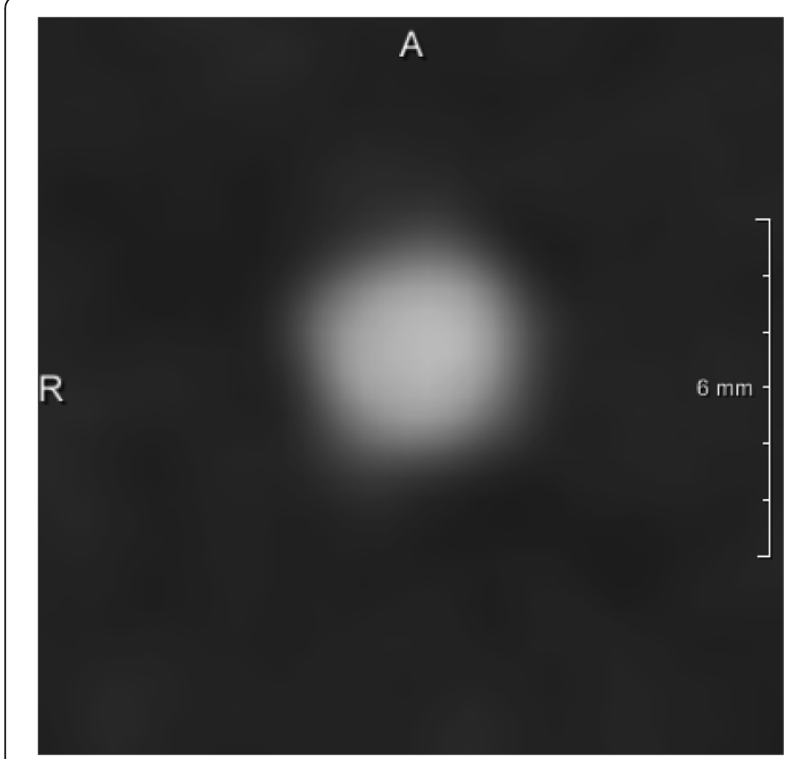

Figure 3 Low calcified stenosis $\left(197 \mathrm{mmHA} / \mathrm{cm}^{3}\right)$ at the same position as Figure 8 ; the stenosis grade is not distinguishable due to insufficient contrast.

$1 \mathrm{~mm}$ (25\%) and $2 \mathrm{~mm}$ (50\%). The movement protocol was derived from an electron beam scan (Figure 2) [9]. The coronary simulator provides an ECG output mapping the movement pattern to an ECG signal readable by the scanner's ECG analysis module.

\section{CT examination protocols}

A 128 slice high pitch capable dual source computed tomography scanner (Siemens Somatom Flash, Siemens Healthcare, Forchheim, Germany) was used for all experiments. The high pitch scans were performed using a dedicated high pitch cardiac protocol (dual source, 100 and $120 \mathrm{kV}, 320 \mathrm{mAs} / \mathrm{rot}$, pitch 3.4, prospective ECG trigger, collimation $128 \times 0.6 \mathrm{~mm}$, FoV $190 \times 190 \mathrm{~mm}$, scan length $90.0 \mathrm{~mm}$, rotation time $0.28 \mathrm{~s}$ ) and a retrospectively ECG gated protocol (100 and $120 \mathrm{kV}, 320$ $\mathrm{mAs} /$ rot, retrospective ECG gating after pulsing at 50$100 \%$, collimation $128 \times 0.6 \mathrm{~mm}$, pitch 0.19 , rotation time $0.28 \mathrm{~s}$ ). Reconstructions were performed according to the vendor's specifications, identically for both modes with B26f, B46f, B70f standard kernels and iterative I26f and I70f kernels with a field of view of $190 \times 190 \mathrm{~mm}$ and slice thicknesses of $0.6 \mathrm{~mm}$ (B26f, B46f, B70f) and $0.75 \mathrm{~mm}$ (I26f, I70f) avoiding undersampling of the acquired data.

\section{Measurements}

Measurements were performed manually using dedicated DICOM viewer software (Synedra View, Version 3.1, Synedra GmbH, Aachen, Germany) by one observer, and checked for validity by two others with 3 and 5 years of experience in thoracic imaging. For each combination of Kernel/Method/kV/plaque phantom three repeated measurements of plaque diameter and three repeated measurements of total vessel diameter were

Table 1 Absolute calcified stenosis diameters (true diameters: 12.5\%: $0.5 \mathrm{~mm}, 25 \%: 1.0 \mathrm{~mm}, 50 \%: 2.0 \mathrm{~mm}$ ), measured total vessel diameters (true diameter $4.0 \mathrm{~mm}$ ) as measured for the different protocol types per phantom type

\begin{tabular}{|c|c|c|c|c|c|}
\hline Density $\left[\mathrm{mmHA} / \mathrm{cm}^{3}\right]$ & True stenosis size & Method & Plaque diameter [mm] & Vessel diameter [mm] & Measured stenosis [\%] \\
\hline \multirow[t]{6}{*}{197} & $12,5 \%$ & High pitch & - & - & - \\
\hline & $(0.5 \mathrm{~mm} / 4.0 \mathrm{~mm})$ & Gating & - & - & - \\
\hline & $25 \%$ & High pitch & - & - & - \\
\hline & $(1.0 \mathrm{~mm} / 4.0 \mathrm{~mm})$ & Gating & - & - & - \\
\hline & $50 \%$ & High pitch & - & - & - \\
\hline & $(2.0 \mathrm{~mm} / 4.0 \mathrm{~mm})$ & Gating & - & - & - \\
\hline \multirow[t]{6}{*}{401} & $12,5 \%$ & High pitch & $0.97 \pm 0.1$ & $3.96 \pm 0.1$ & $24.6 \pm 2.7$ \\
\hline & $(0.5 \mathrm{~mm} / 4.0 \mathrm{~mm})$ & Gating & $0.88 \pm 0.1$ & $3.95 \pm 0.1$ & $22.4 \pm 3.3$ \\
\hline & $25 \%$ & High pitch & $1.48 \pm 0.2$ & $4.13 \pm 0.2$ & $35.8 \pm 4.3$ \\
\hline & $(1.0 \mathrm{~mm} / 4.0 \mathrm{~mm})$ & Gating & $1.40 \pm 0.2$ & $4.00 \pm 0.1$ & $34.9 \pm 4.6$ \\
\hline & $50 \%$ & High pitch & $1.98 \pm 0.2$ & $4.08 \pm 0.2$ & $48.6 \pm 4.3$ \\
\hline & $(2.0 \mathrm{~mm} / 4.0 \mathrm{~mm})$ & Gating & $1.98 \pm 0.2$ & $4.00 \pm 0.1$ & $49.6 \pm 4.6$ \\
\hline \multirow[t]{6}{*}{796} & $12,5 \%$ & High pitch & $1.27 \pm 0.1$ & $4.35 \pm 0.4$ & $29.2 \pm 2.4$ \\
\hline & $(0.5 \mathrm{~mm} / 4.0 \mathrm{~mm})$ & Gating & $1.18 \pm 0.1$ & $4.52 \pm 0.4$ & $26.2 \pm 2.6$ \\
\hline & $25 \%$ & High pitch & $1.80 \pm 0.4$ & $4.52 \pm 0.4$ & $39.7 \pm 5.9$ \\
\hline & $(1.0 \mathrm{~mm} / 4.0 \mathrm{~mm})$ & Gating & $1.71 \pm 0.4$ & $4.70 \pm 0.5$ & $36.1 \pm 5.0$ \\
\hline & $50 \%$ & High pitch & $2.39 \pm 0.4$ & $3.99 \pm 0.3$ & $59.6 \pm 7.1$ \\
\hline & $(2.0 \mathrm{~mm} / 4.0 \mathrm{~mm})$ & Gating & $2.22 \pm 0.3$ & $3.93 \pm 0.2$ & $56.3 \pm 5.9$ \\
\hline
\end{tabular}

The lowest density plaque phantoms $\left(197 \mathrm{mmHA} / \mathrm{cm}^{3}\right)$ were not measurable as no sufficient contrast could be established between the lumen and the plaque mimic. 
performed resulting in a total of 2,880 data points. The repeated measurements were averaged and used for statistical analyses.

\section{Statistical analyses}

Statistical analysis was performed using SPSS 19 (SPSS Inc., Chicago, Illinois, USA) and MedCalc (MedCalc Software, Mariakerke, Belgium). Tests performed were Analysis of Variances with post-hoc testing and BlandAltman as well as Mountain plot method comparisons. P-values of 0.05 or lower were considered statistically significant, multiple testing correction (Bonferroni) was performed where applicable. Additional tests were performed using non-parametric Mann-Whitney-U analyses.

\section{Results}

The plaque phantom featuring the lowest calcification level $\left(197 \mathrm{mmHA} / \mathrm{cm}^{3}\right)$ was not measureable with the applied methods due to low contrast between plaque and vessel lumen (Figure 3). The following data result from the measurements of the intermediately $\left(401 \mathrm{mmHA} / \mathrm{cm}^{3}\right)$ and heavily $\left(796 \mathrm{mmHA} / \mathrm{cm}^{3}\right)$ calcified plaque phantoms.

\section{Comparison to phantom dimensions}

In comparison to the phantom vessel dimensions retrospectively ECG-gated and prospectively ECG-triggered high pitch scanning provided vessel diameters of between $3.93 \pm$ $0.2 \mathrm{~mm}$ and $4.70 \pm 0.5 \mathrm{~mm}$ with a tendency to a slight
Table 3 Average difference in degree of stenosis $(\Delta$ percentage) and measured plaque diameter ( $\Delta$ diameter) between the two cardiac CT methods and tube voltages (first vs. second mentioned)

\begin{tabular}{lll}
\hline Comparison & $\boldsymbol{\Delta}$ Percentage & $\boldsymbol{\Delta}$ Diameter \\
\hline Gated/high pitch & $2 \pm 8 \mathrm{PP}$ & $0.09 \pm 0.31 \mathrm{~mm}$ \\
$100 \mathrm{kV} / 120 \mathrm{kV}$ & $3 \pm 8 \mathrm{PP}$ & $0.14 \pm 0.32 \mathrm{~mm}$ \\
\hline
\end{tabular}

(PP: percent points).

overestimation (Table 1). The plaque thickness was measured at between $0.88 \pm 0.1 \mathrm{~mm}$ and $1.27 \pm 0.1 \mathrm{~mm}$ $(0.5 \mathrm{~mm} / 12.5 \%), 1.40 \pm 0.2 \mathrm{~mm}$ and $1.80 \pm 0.4 \mathrm{~mm}(1 \mathrm{~mm} /$ $25 \%)$ and $1.98 \pm 0.2 \mathrm{~mm}$ and $2.39 \pm 0.4 \mathrm{~mm}(2 \mathrm{~mm} / 50 \%)$ again showing an overestimation for both cardiac CT techniques which was more pronounced for high-pitch scanning. These findings led to the following degrees of stenoses: $22.4 \pm 3.3 \%$ and $29.2 \pm 2.4 \%$ (12.5\% stenoses), $34.9 \pm 4.6 \%$ and $39.7 \pm 5.9 \%$ (25\% stenoses) and $48.6 \pm 4.3 \%$ and $59.6 \pm 7.1 \%$ ( $50 \%$ stenoses) for retrospectively gated and prospectively triggered high pitch, respectively.

Separated by heart rate of the phantom, both examination protocols showed an overestimation of vessel diameter, plaque diameter and degree of stenosis which was more pronounced for the $12.5 \%$ in comparison to the $50 \%$ stenosis and for the high pitch protocol in comparison to ECG-gated scanning. Diameter measurements for the same degree of stenosis at different heart rates did not show relevant differences (Table 2).

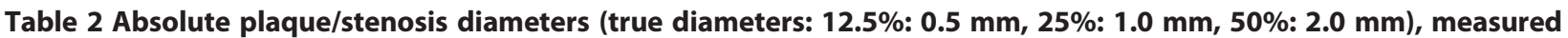
vessel diameters (true diameter $4.0 \mathrm{~mm}$ ) per heart rate and cardiac CT method

\begin{tabular}{|c|c|c|c|c|c|c|}
\hline True stenosis [\%] & Heart rate [bpm] & Method & Plaque diameter [mm] & Vessel diameter [mm] & Stenosis [\%] & Deviation [PP] \\
\hline \multirow[t]{6}{*}{$12.5 \%$} & 50 & High pitch & $1.13 \pm 0.19$ & $4.17 \pm 0.35$ & $27.0 \pm 3.5$ & 14.5 \\
\hline & & Gating & $1.03 \pm 0.19$ & $4.23 \pm 0.45$ & $24.4 \pm 3.7$ & 11.9 \\
\hline & 70 & High pitch & $1.12 \pm 0.18$ & $4.14 \pm 0.35$ & $27.1 \pm 3.4$ & 14.6 \\
\hline & & Gating & $1.03 \pm 0.19$ & $4.26 \pm 0.41$ & $24.1 \pm 3.6$ & 11.6 \\
\hline & 90 & High pitch & $1.11 \pm 0.19$ & $4.15 \pm 0.30$ & $26.7 \pm 3.5$ & 14.2 \\
\hline & & Gating & $1.03 \pm 0.19$ & $4.22 \pm 0.40$ & $24.4 \pm 3.4$ & 11.9 \\
\hline \multirow[t]{6}{*}{$25 \%$} & 50 & High pitch & $1.63 \pm 0.34$ & $4.35 \pm 0.38$ & $37.2 \pm 5.7$ & 12.2 \\
\hline & & Gating & $1.53 \pm 0.33$ & $4.35 \pm 0.49$ & $35.0 \pm 4.6$ & 10.0 \\
\hline & 70 & High pitch & $1.65 \pm 0.33$ & $4.33 \pm 0.36$ & $37.8 \pm 5.1$ & 12.8 \\
\hline & & Gating & $1.53 \pm 0.34$ & $4.35 \pm 0.51$ & $34.8 \pm 4.6$ & 9.8 \\
\hline & 90 & High pitch & $1.66 \pm 0.35$ & $4.29 \pm 0.39$ & $38.4 \pm 5.8$ & 13.4 \\
\hline & & Gating & $1.61 \pm 0.36$ & $4.36 \pm 0.51$ & $36.7 \pm 5.2$ & 11.7 \\
\hline \multirow[t]{6}{*}{$50 \%$} & 50 & High pitch & $2.19 \pm 0.37$ & $4.06 \pm 0.20$ & $53.9 \pm 8.0$ & 3.9 \\
\hline & & Gating & $2.08 \pm 0.28$ & $3.95 \pm 0.18$ & $52.7 \pm 6.2$ & 2.7 \\
\hline & 70 & High pitch & $2.16 \pm 0.36$ & $4.00 \pm 0.23$ & $54.0 \pm 7.9$ & 4.0 \\
\hline & & Gating & $2.11 \pm 0.27$ & $3.99 \pm 0.14$ & $52.9 \pm 6.1$ & 2.9 \\
\hline & 90 & High pitch & $2.20 \pm 0.38$ & $4.05 \pm 0.21$ & $54.4 \pm 8.4$ & 4.4 \\
\hline & & Gating & $2.11 \pm 0.32$ & $3.96 \pm 0.19$ & $53.1 \pm 6.7$ & 3.1 \\
\hline
\end{tabular}




\section{Comparison of trigger types}

High pitch scanning resulted in a larger depiction of the plaques in comparison to the ECG-gated scan method $(0.09 \pm 0.31 \mathrm{~mm})$. This difference accounted for an additional overestimation of the stenoses by $2 \pm 8$ percent points (PP) (Table 3). Bland-Altman plotting (Figure 4) revealed this systematic difference, while mountain plotting additionally revealed no outliers and a narrow distribution profile.

\section{Trigger type and heart rate}

Comparing the two CT imaging methods with respect to their plaque depiction depending on heart rate, a descending difference of the measured plaque diameter was found with increasing heart rate $(50 \mathrm{bpm}: 0.1 \pm 0.28 \mathrm{~mm}$, $70 \mathrm{bpm}: 0.09 \pm 0.31 \mathrm{~mm}$ and $90 \mathrm{bpm}: 0.07 \pm 0.35 \mathrm{~mm}$, Table 4). However, the difference was so small at all heart rates that no clinically relevant effect on the degree of stenosis (2 $\pm 7-8 \mathrm{PP})$ was observed (Table 4$)$.

\section{Tube voltage}

In comparison to $120 \mathrm{kV}$ tube voltage $100 \mathrm{kV} \mathrm{CT}$ imaging resulted in a difference of $0.14 \pm 0.32 \mathrm{~mm}$ regarding plaque diameters. This led to a $3 \pm 8 \mathrm{PP}$ overestimation of the stenosis diameter using $100 \mathrm{kV}$ scan protocols (Figure 5, Table 4).

\section{Reconstruction kernels}

Smooth reconstruction kernels (B26f, I26f) showed a higher deviation from the true plaque diameters in comparison to the sharp kernels (B70f, I70f). This result was even more pronounced for the heavily calcified plaques which were significantly overestimated regarding their diameter when smooth kernels were used for image reconstruction. The B46f kernel exhibited a intermediate
Table 4 Average difference in degree of stenosis ( $\Delta$ percentage) and measured plaque diameter ( $\Delta$ diameter) per heart rate between the two cardiac CT examination protocols (PP: percent points)

\begin{tabular}{lll}
\hline Heart rate & $\boldsymbol{\Delta}$ Percentage (gating vs. high pitch) & $\boldsymbol{\Delta}$ Diameter \\
\hline $50 \mathrm{bpm}$ & $2 \pm 7 \mathrm{PP}$ & $0.10 \pm 0.28 \mathrm{~mm}$ \\
$70 \mathrm{bpm}$ & $2 \pm 8 \mathrm{PP}$ & $0.09 \pm 0.31 \mathrm{~mm}$ \\
$90 \mathrm{bpm}$ & $2 \pm 8 \mathrm{PP}$ & $0.07 \pm 0.35 \mathrm{~mm}$ \\
\hline
\end{tabular}

performance, with the least deviation in the less calcified plaque and a deviation between the standard and iterative kernels in the heavily calcified setting. The comparison between corresponding standard and iterative reconstruction kernels (B26f vs. I26f; B70f vs. I70f) did not reveal any relevant difference (Table 5, Figure 6).

\section{Radiation dose comparison}

Radiation doses were significantly lower for high pitch CT in comparison to ECG gated scanning (258.0 mGy vs. $2829.8 \mathrm{mGy}$ for CTDIvol, $36.3 \mathrm{mGycm}$ vs. 341.2 mGycm for the dose-length-product, DLP), and lower for $100 \mathrm{kV}$ vs. $120 \mathrm{kV}$ scan protocols (962.6 mGy vs. $2125.2 \mathrm{mGy}$ CTDIvol and $115.1 \mathrm{mGycm}$ vs $262.4 \mathrm{mGycm}$ DLP). All these differences were statistically significant $(\mathrm{p} \leq 0.0001$ for trigger type and $\mathrm{p} \leq 0.0005$ for tube voltage, Tables 6, 7).

\section{Motion artifacts}

Coronal reconstruction revealed motion artifacts, present at the proximal end of the coronal phantom for the $90 \mathrm{bpm}$ high pitch prospectively gated scans (Figure 7), which were not present in retrospectively gated scanning or the 50 or $70 \mathrm{bpm}$ high pitch experiments. No other occurrences of motion artifacts were observed.
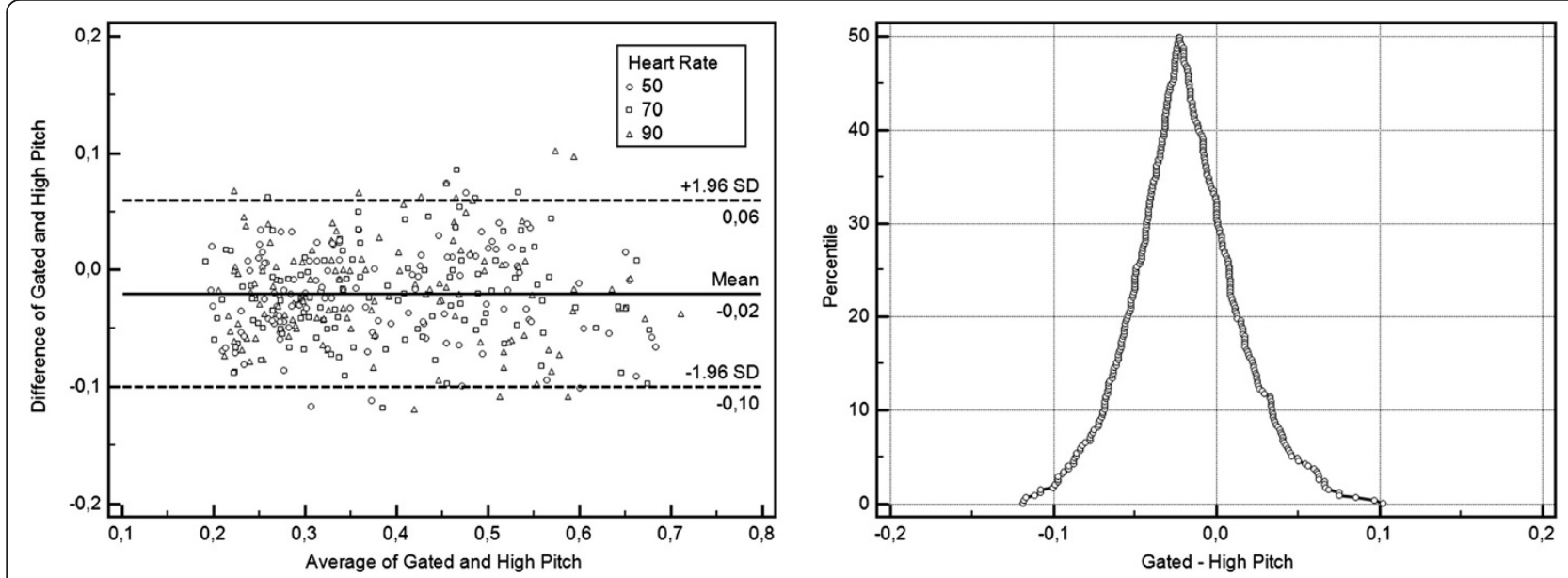

Figure 4 Method comparisons for the two trigger types under investigation. Bland-Altman-Plotting reveals a slight overestimation of stenosis percentage $(2 \pm 8 \%)$. Mountain plotting shows no relevant outliers. 

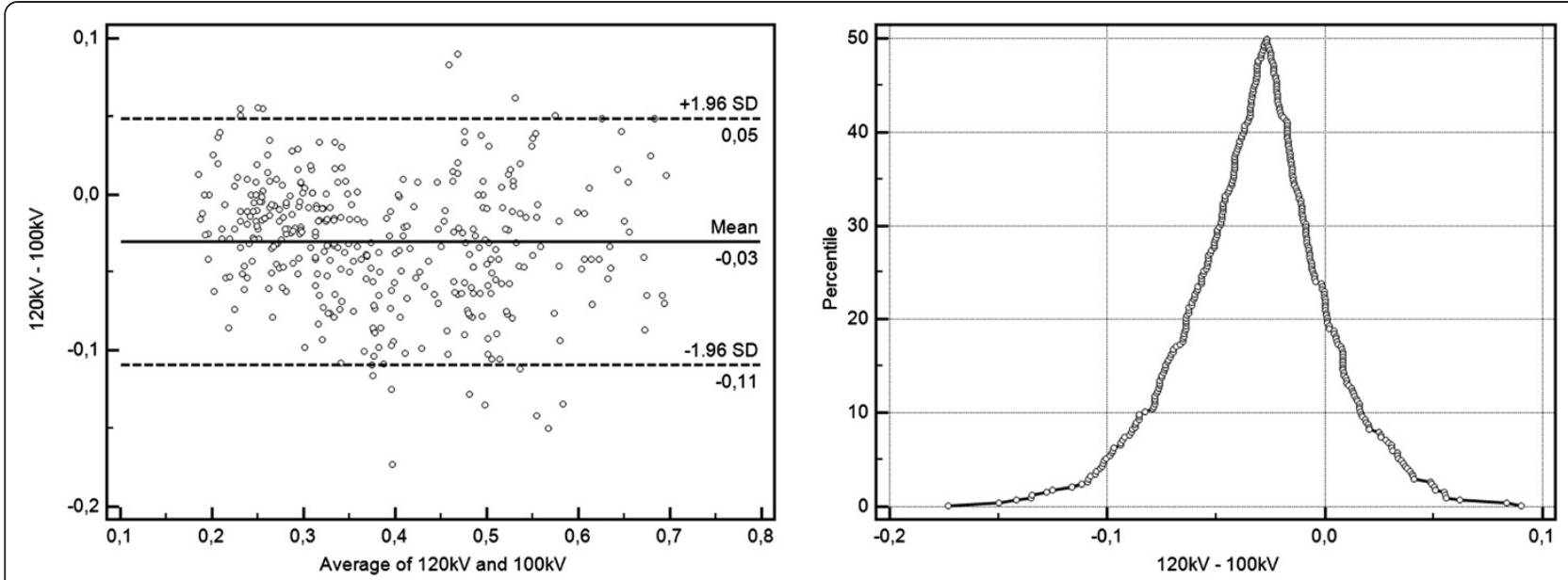

Figure $\mathbf{5}$ Method comparison plots for the tube voltage comparison at $\mathbf{1 0 0}$ and $\mathbf{1 2 0} \mathbf{k V}$. While on average only a slight overestimation for $100 \mathrm{kV}$ is shown, some outliers are present in mountain plotting.

\section{Discussion}

Despite the fact that coronary CTA has found its way into the clinical workup of patients with suspected coronary artery disease, there are still major concerns regarding the applied radiation dose, which was determined to be in the typically order of $12 \mathrm{mSv}(8-18 \mathrm{mSv})$ [10]. In comparison the effective radiation doses applied during invasive coronary angiography are reported to be approximately $5 \mathrm{mSv}$ [11] with ranges from 2.3 to $22.7 \mathrm{mSv}$ [3]. Because of its ability to rule out hemodynamically relevant coronary stenoses with a high negative predictive value, coronary CTA is especially suited for patients presenting with typical symptoms but having a low pre-test likelihood for coronary artery disease [12]. During the last few years different technical developments have been introduced in clinical routine practice in order to significantly reduce radiation dose of coronary CTA. These techniques include the use of lower tube voltage (e.g. 100 instead of $120 \mathrm{kV}$ ) [13] and the application of ECG-dependent tube current modulation. The latter bases upon the reduction of tube current of up to $80 \%$ during systolic phase and full dose is only applied during diastolic phase. This approach allows for dose reduction of up to
$50 \%$ [14]. Another technique resulting in substantial reduction of radiation dose is realized by the use of prospective ECG-triggered transverse data acquisition (also known as "step and shot acquisition") instead of retrospective gated spiral scanning [15].

With the introduction of a modern dual-source CT scanner with simultaneous acquisition of 64 slices and a gantry rotation time of $330 \mathrm{~ms}$ a temporal resolution of $83 \mathrm{~ms}$ became technically feasible [16]. Latest generation of dual-source scanners reduced the rotation time to $0.28 \mathrm{~s}$. Furthermore, due to the ca. $95^{\circ}$ offset of both $\mathrm{x}$ ray tubes within the gantry of dual source $\mathrm{CT}$ in combination with fast and precise table movement the use of high-pitch (>3.0) scan protocols for coronary CTA could be performed. The result was the ability to cover the whole heart within a single cardiac cycle [17]. Since then, different studies showed the diagnostic value of prospectively ECG-triggered high-pitch spiral coronary CTA $[18,19]$.

The aim of the presented study was to investigate influence of scan protocol in combination with ECGsynchronization (prospective triggered high-pitch vs. retrospective ECG-gated spiral CT), tube voltage (100

Table 5 Pairwise comparisons of the average difference in degree of stenosis ( $\Delta$ percentage) and measured plaque diameter for all used kernels

\begin{tabular}{lrrrr}
\hline & $\mathbf{7 9 6} \mathbf{~ m m H A} / \mathbf{c m}^{\mathbf{3}}$ & & $\mathbf{4 0 1} \mathbf{~ m m H A} / \mathbf{c m}^{\mathbf{3}}$ & $\boldsymbol{\Delta}$ \\
\hline B26f & $\boldsymbol{\Delta}$ Diameter & $\boldsymbol{\Delta}$ Percentage & $0.39 \pm 0.27 \mathrm{~mm}$ & $\mathbf{\Delta}$ Percentage \\
I26f & $0.88 \pm 0.26 \mathrm{~mm}$ & $15.9 \pm 4.2 \%$ & $0.38 \pm 0.26 \mathrm{~mm}$ & $4.6 \pm 6.8 \%$ \\
B46f & $0.86 \pm 0.25 \mathrm{~mm}$ & $10.8 \pm 5.0 \%$ & $0.18 \pm 0.26 \mathrm{~mm}$ & $6.9 \pm 6.9 \%$ \\
B70f & $0.53 \pm 0.27 \mathrm{~mm}$ & $9.2 \pm 6.6 \%$ & $0.26 \pm 0.27 \mathrm{~mm}$ & $8.5 \pm 6.6 \%$ \\
I70f & $0.36 \pm 0.33 \mathrm{~mm}$ & $15.3 \pm 4.0 \%$ & $0.21 \pm 0.26 \mathrm{~mm}$ & $5.8 \pm 6.6 \%$ \\
\hline
\end{tabular}



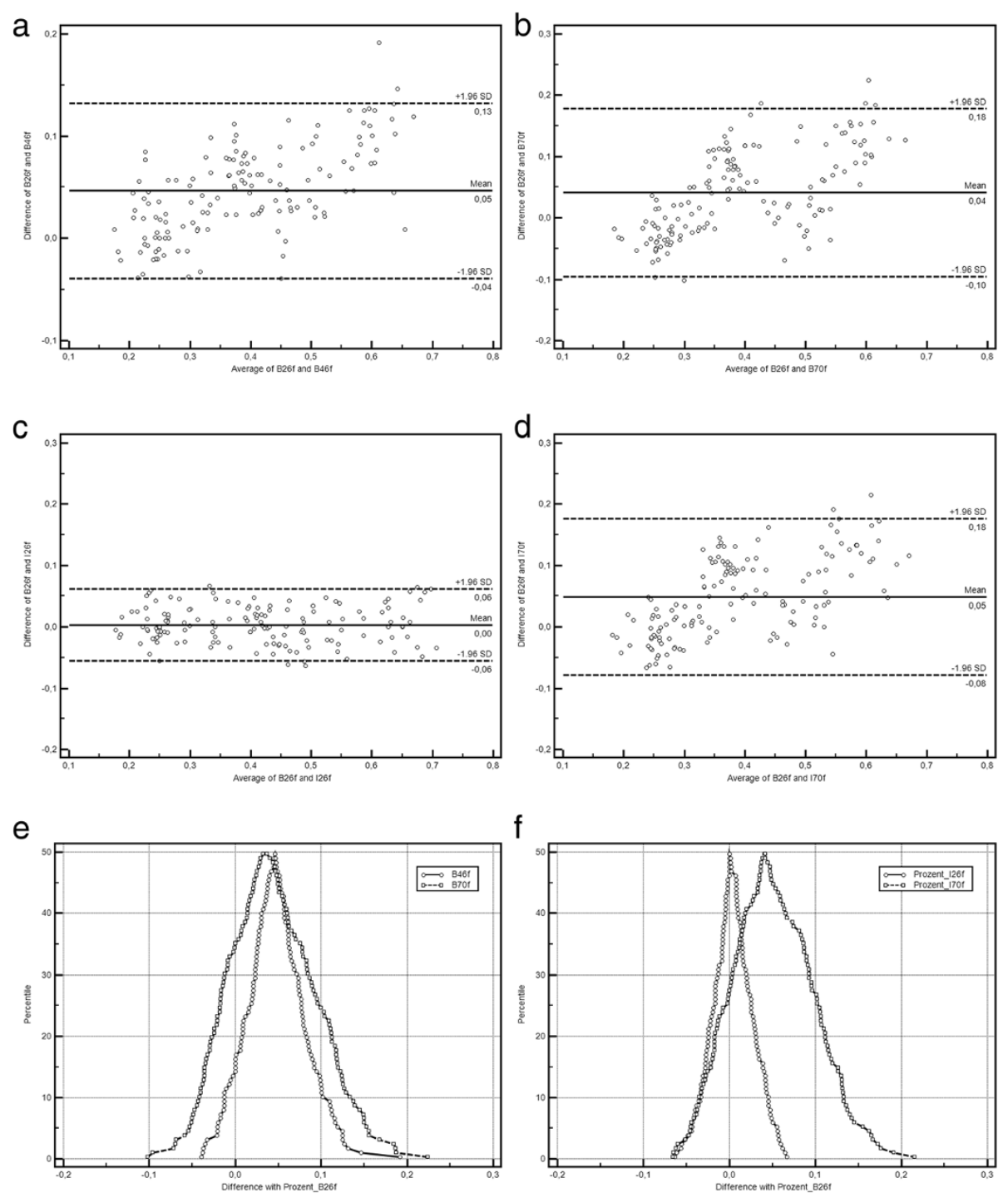

Figure 6 Method comparison plots for kernel comparisons (a-d Bland-Altman plots, e-f mountain plots). Shown are the differences between the standard B26f and the other kernels under investigation (a, e: B46f, b, e: B70f, c, f: 126f, d, f: 170f). While for B26f and $126 \mathrm{f}$ align perfectly, skewed distributions with outliers are visible for all other comparisons.

vs. $120 \mathrm{kV})$ and heart rate $(50,70,90 \mathrm{bpm})$ on quantification of coronary artery stenosis due to plaques with different calcification levels. In general, all used CT examination protocols resulted in an overestimation of stenoses which increased with increasing calcium content and decreased with increasing grade of stenoses. This overestimation was slightly higher for the high pitch examination protocols in comparison to the retrospective ECG-gated technique. Both observations (overestimation of small lesions, which are highly calcified) are mainly due to blooming artifacts which occur when sharp edges with high attenuation differences are encountered in the scan volume [20,21]. A reason for the slight worsening of this phenomenon, when high pitch examination protocols are used, may be the fact that attenuation data of two separate detectors are used for image reconstruction. However, in the clinical situation this finding may be of little relevance due to a maximum difference of 3 percent points regarding grade of stenosis (small, highly calcified plaque).

In most of the recently published clinical studies on prospective ECG-triggered high pitch cardiac $\mathrm{CT}$ a heart rate more than $60 \mathrm{bpm}$ was defined as an exclusion criterion $[4,18]$. In our experimental setup there were no significant differences regarding vessel diameter, plaque diameter and grade of stenoses between the different heart rates $(50,70$, 90) although we found a slight trend to larger deviations of the measured parameters comparing the 50 and the $90 \mathrm{bpm}$ data (Table 2). The motion artifacts observed at 
Table 6 Dose comparison between the two used cardiac CT methods

\begin{tabular}{lrrr}
\hline Trigger & Gated & High pitch & Statistics \\
\hline CTDlvol $[\mathrm{mGy}]$ & $2829.8 \pm 1539.4$ & $258.0 \pm 66.3$ & $\mathrm{p}<0.0001$ \\
DLP [mGycm] & $341.2 \pm 150.7$ & $36.3 \pm 9.6$ & $\mathrm{p}<0.0001$
\end{tabular}

Both DLP and CTDIvol are significantly lower for high pitch cardiac CT protocols in comparison to gating.

90 bpm high pitch scanning should however raise concerns about the applicability of the technique at such heart rates (Figure 8). This finding is at least to some degree in contrast to clinical findings which were recently published [22]. Scharf et al. reported about a cohort of 111 consecutive patients who underwent prospectively ECG-triggered high-pitch spiral CT of the chest for non-cardiac reason. The evaluation of image quality showed a significant difference of mean heart rate and mean heart rate variability between patients with diagnostic and non-diagnostic images of the coronary arteries. The optimal values were calculated with a heart rates lower than $64 \mathrm{bpm}$ and heart rate variabilities of less than $13 \mathrm{bpm}$. With respect to this result it needs to be discussed that we used an extremely reliable experimental setup providing an absolutely stable heart rate. Nevertheless, our results may be a hint that prospectively ECG-triggered high-pitch cardiac CT may also be suited for patients presenting stable heart rates $>60 \mathrm{bpm}$ and a low heart rate variability. This hypothesis is supported by the results of Feuchtner et al. who found significantly higher diagnostic image quality in patients with stable sinus rhythm but without premedication for heart rate control in comparison to patients with arrhythmia using a prospective triggered "step and shoot" CT-examination mode [23]. In this study a mean heart rate of $66.2 \pm 8 \mathrm{bpm}$ in patient group with stable sinus rhythm was associated with only $0.5 \%$ nondiagnostic coronary segments whereas $4 \%$ of coronary segments were scored non-diagnostic in the arrhythmia group with a mean heart rate of $70 \pm 15 \mathrm{bpm}$.

In our experimental setting the use of a prospectively ECG-triggered high-pitch spiral examination protocol resulted in a reduction of radiation exposure of approximately $90 \%$ in comparison to a retrospective ECG-gated cardiac CT-examination (Table 6). This finding is mainly due to the significant oversampling using a pitch of 0.19 for retrospective ECG-gating in order to acquire enough data in all

Table 7 Dose comparison between the two used tube voltages

\begin{tabular}{lrrr}
\hline $\mathbf{k V}$ & $\mathbf{1 0 0}$ & $\mathbf{1 2 0}$ & Statistics \\
\hline CTDlvol [mGy] & $962.6 \pm 862.9$ & $2125.2 \pm 2082.3$ & $\mathrm{p}=0.0005$ \\
DLP [mGycm] & $115.1 \pm 93.4$ & $262.4 \pm 225.2$ & $\mathrm{p}=0.0002$ \\
\hline
\end{tabular}

Both DLP and CTDlvol are significantly lower for $100 \mathrm{kV}$ in comparison to $120 \mathrm{kV}$.

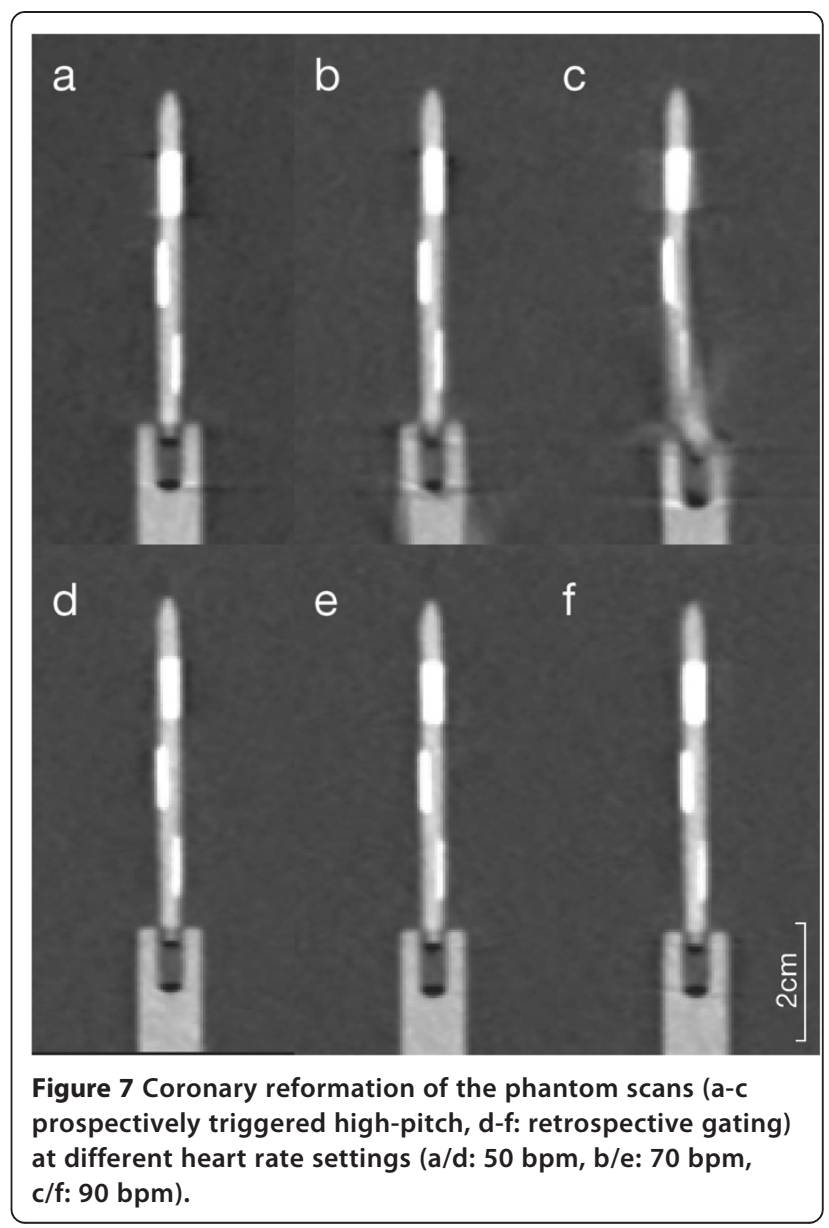

cardiac phases. On the other hand this allows quantification of cardiac function in addition to the evaluation of coronary arteries. Comparing the measured diameters for the 100 and $120 \mathrm{kV}$ examination modes we found a slight overestimation for the $100 \mathrm{kV}$ CT-protocol (Table 3). However, we consider the differences $(3 \pm 8 \%)$ clinically not relevant whereas reduction of tube voltage results in further reduction of radiation dose applied to the patient. Due to the fact that lower tube voltage leads to increased image noise some authors used $100 \mathrm{kV}$ scan protocols in patients $<100 \mathrm{~kg}$ body weight and $120 \mathrm{kV}$ for $>100 \mathrm{~kg}$, respectively [24].

Due to the experimental design of the study our results cannot be directly transferred into the clinical situation. The employed phantom provides a stable sinus rhythm without any variability due to arrhythmia or respiration. Furthermore, motion of the patient is not an issue in this setup. Another limitation that needs to be discussed is the fact that we did not really measure the applied radiation dose but simply compared the dose indices derived from the CT-scanner's software. Moreover, regarding the different tube voltages (100 vs. $120 \mathrm{kV}$ ) we cannot make conclusion regarding image quality in obese patients due to 


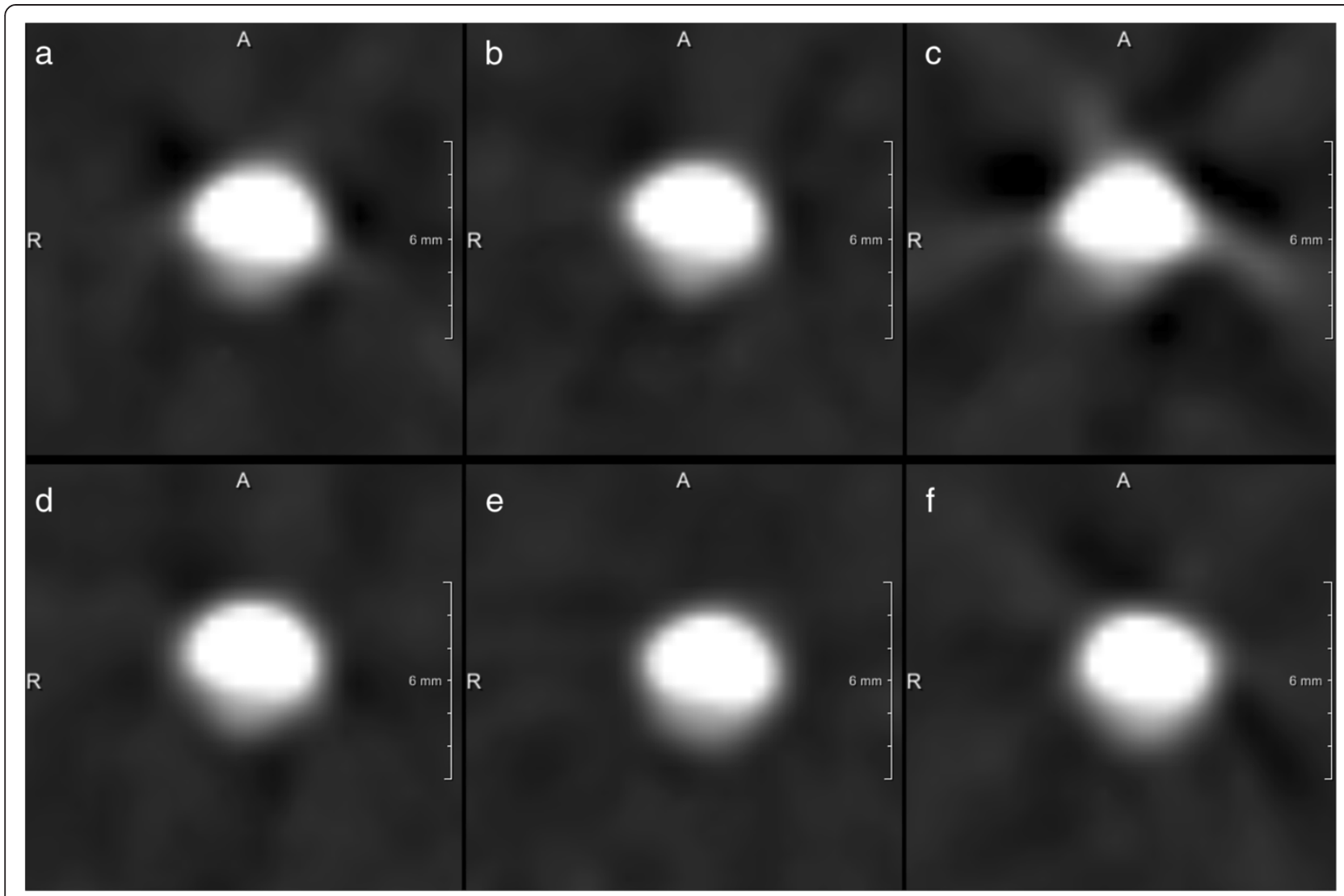

Figure 8 Image data reconstructed in different heart rate settings (a/d: 50 bpm; b/e: 70 bpm; c/f: 90 bpm, B26f) using either prospectively ECG-triggered high-pitch scanning $(a-c)$ or retrospectively ECG-gated cardiac CT (d - f).

the fact that the phantom does not account for different subject weight. Further experiments should include these factors, for instance by applying different attenuation phantoms in the setup.

\section{Conclusion}

In conclusion, the presented results do not reveal any relevant differences in vessel diameter, plaque diameter and grade of stenoses between prospectively ECG-triggered high-pitch spiral CT in comparison to retrospectively ECGgated spiral data acquisition in differently calcified plaques using a motion phantom. While there was reasonable agreement even at higher heart rates $(90 \mathrm{bpm})$ between both examination modes, the presence of motion artifacts at $90 \mathrm{bpm}$ questions the full applicability of the high pitch technique under these circumstances.

\section{Competing interests}

The study was carried out as part of a research agreement with Siemens Healthcare - CT Division -, Forchheim, Germany. TA is an employee of Siemens Healthcare, Forchheim, Germany.

\section{Authors' contributions}

Study design: TP, ED, PI, TA, CKK, AHM, PB, experiments, measurements: TP, $E D, P I, T A, P B$, manuscript draft: TP, PI, TA, CKK, AHM, PB. All authors read and approved the final manuscript.

\section{Author details}

'Department of Diagnostic and Interventional Radiology, Aachen University Hospital, RWTH Aachen University, Pauwelsstr. 30, 52074 Aachen, Germany.

${ }^{2}$ Surgical Planning Laboratory, Department of Radiology, Brigham and Women's Hospital, 75 Francis Street, 02115 Boston, USA. ${ }^{3}$ Applied Medical Engineering, Helmholtz-Institute Aachen, RWTH Aachen University, Pauwelsstr. 20, 52074 Aachen, Germany. ${ }^{4}$ Siemens Healthcare, CT Division, Forchheim, Germany. ${ }^{5}$ Department of Diagnostic and Interventional Radiology, University Hospital Marburg, Philipps University of Marburg, Marburg, Germany.

Received: 26 April 2013 Accepted: 3 August 2014

Published: 1 September 2014

\section{References}

1. Vorobiof G, Achenbach $\mathrm{S}$, Narula J: Minimizing radiation dose for coronary CT angiography. Cardiol Clin 2012, 30(1):9-17.

2. Alkadhi $\mathrm{H}$ : Radiation dose of cardiac $\mathrm{CT}$-what is the evidence? Eur Radio 2009, 19(6):1311-1315.

3. Einstein AJ, Moser KW, Thompson RC, Cerqueira MD, Henzlova MJ: Radiation dose to patients from cardiac diagnostic imaging. Circulation 2007, 116(11):1290-1305.

4. Achenbach S, Marwan M, Ropers D, Schepis T, Pflederer T, Anders K, Kuettner A, Daniel WG, Uder M, Lell MM: Coronary computed tomography angiography with a consistent dose below $1 \mathrm{mSv}$ using prospectively electrocardiogram-triggered high-pitch spiral acquisition. Eur Heart J 2010, 31(3):340-346.

5. Flohr TG, Leng S, Yu L, Allmendinger T, Bruder H, Petersilka M, Eusemann CD, Stierstorfer K, Schmidt B, McCollough CH: Dual-source spiral CT with pitch up to 3.2 and $75 \mathrm{~ms}$ temporal resolution: image reconstruction and assessment of image quality. Med Phys 2009, 36(12):5641-5653. 
6. Flohr TG, McCollough CH, Bruder H, Petersilka M, Gruber K, Suss C, Grasruck M, Stierstorfer K, Krauss B, Raupach R, Primak AN, Kuttner A, Achenbach S, Becker C, Kopp A, Ohnesorge BM: First performance evaluation of a dual-source CT (DSCT) system. Eur Radiol 2006, 16(2):256-268.

7. Morsbach F, Gordic S, Desbiolles L, Husarik D, Frauenfelder T, Schmidt B, Allmendinger T, Wildermuth S, Alkadhi $\mathrm{H}$, Leschka S: Performance of turbo high-pitch dual-source $\mathrm{CT}$ for coronary $\mathrm{CT}$ angiography: first ex vivo and patient experience. Eur Radiol 2014, 24(8):1889-1895.

8. Gordic S, Husarik DB, Desbiolles L, Leschka S, Frauenfelder T, Alkadhi H: High-pitch coronary CT angiography with third generation dual-source CT: limits of heart rate. Int J Cardiovasc Imaging 2014, 30(6):1173-1179.

9. Ulzheimer S, Kalender WA: Assessment of calcium scoring performance in cardiac computed tomography. Eur Radiol 2003, 13(3):484-497.

10. Hausleiter J, Meyer T, Hermann F, Hadamitzky M, Krebs M, Gerber TC, McCollough C, Martinoff S, Kastrati A, Schomig A, Achenbach S: Estimated radiation dose associated with cardiac CT angiography. JAMA 2009, 301(5):500-507.

11. Coles DR, Smail MA, Negus IS, Wilde P, Oberhoff M, Karsch KR, Baumbach A: Comparison of radiation doses from multislice computed tomography coronary angiography and conventional diagnostic angiography. J Am Coll Cardiol 2006, 47(9):1840-1845.

12. Hoffman U, Venkatesh V, White RD, Woodard PK, Carr JJ, Dorbala S, Earls JP, Jacobs JE, Mammen L, Martin ET, Ryan T, White CS: ACR Appropriateness Criteria: Acute Nonspecific Chest Pain - low Probability of Coronary Artery Disease. Reston, VA, USA: American College of Radiology; 2011. ACR Appropriateness Criteria.

13. Pflederer T, Rudofsky L, Ropers D, Bachmann S, Marwan M, Daniel WG, Achenbach S: Image quality in a low radiation exposure protocol for retrospectively ECG-gated coronary $C T$ angiography. AJR Am J Roentgenol 2009, 192(4):1045-1050.

14. Paul JF, Abada HT: Strategies for reduction of radiation dose in cardiac multislice CT. Eur Radiol 2007, 17(8):2028-2037.

15. Maruyama T, Takada M, Hasuike T, Yoshikawa A, Namimatsu E, Yoshizumi T: Radiation dose reduction and coronary assessability of prospective electrocardiogram-gated computed tomography coronary angiography: comparison with retrospective electrocardiogram-gated helical scan. J Am Coll Cardiol 2008, 52(18):1450-1455.

16. Flohr TG, Bruder $\mathrm{H}$, Stierstorfer $\mathrm{K}$, Petersilka M, Schmidt B, McCollough $\mathrm{CH}$ : Image reconstruction and image quality evaluation for a dual source $C T$ scanner. Med Phys 2008, 35(12):5882-5897.

17. Achenbach S, Marwan M, Schepis T, Pflederer T, Bruder H, Allmendinger T, Petersilka M, Anders K, Lell M, Kuettner A, Ropers D, Daniel WG, Flohr T: High-pitch spiral acquisition: a new scan mode for coronary $C T$ angiography. J Cardiovasc Comput Tomogr 2009, 3(2):117-121.

18. Achenbach S, Goroll T, Seltmann M, Pflederer T, Anders K, Ropers D, Danie WG, Uder M, Lell M, Marwan M: Detection of coronary artery stenoses by low-dose, prospectively ECG-triggered, high-pitch spiral coronary CT angiography. JACC Cardiovasc Imaging 2011, 4(4):328-337.

19. Kropil P, Rojas CA, Ghoshhajra B, Lanzman RS, Miese FR, Scherer A, Kalra M, Abbara S: Prospectively ECG-triggered high-pitch spiral acquisition for cardiac CT angiography in routine clinical practice: initial results. J Thorac Imaging 2012, 27(3):194-201.

20. Dey D, Slomka P, Chien D, Fieno D, Abidov A, Saouaf R, Thomson L, Friedman JD, Berman DS: Direct quantitative in vivo comparison of calcified atherosclerotic plaque on vascular MRI and CT by multimodality image registration. J Magn Reson Imaging 2006, 23(3):345-354.

21. Joseph PM, Spital RD: The exponential edge-gradient effect in x-ray computed tomography. Phys Med Biol 1981, 26(3):473-487.

22. Scharf M, Bink R, May MS, Hentschke C, Achenbach S, Uder M, Lell MM: High-pitch thoracic CT with simultaneous assessment of coronary arteries: effect of heart rate and heart rate variability on image quality and diagnostic accuracy. JACC Cardiovasc Imaging 2011, 4(6):602-609.

23. Feuchtner G, Goetti R, Plass A, Baumueller S, Stolzmann P, Scheffel H, Wieser M, Marincek B, Alkadhi H, Leschka S: Dual-step prospective ECG-triggered 128-slice dual-source CT for evaluation of coronary arteries and cardiac function without heart rate control: a technical note. Eur Radiol 2010, 20(9):2092-2099.

24. Lell M, Marwan M, Schepis T, Pflederer T, Anders $K$, Flohr T, Allmendinger T, Kalender W, Ertel D, Thierfelder C, Kuettner A, Ropers D, Daniel WG, Achenbach S: Prospectively ECG-triggered high-pitch spiral acquisition for coronary CT angiography using dual source $\mathrm{CT}$ : technique and initial experience. Eur Radiol 2009, 19(11):2576-2583.

doi:10.1186/1471-2342-14-30

Cite this article as: Penzkofer et al:: Influence of trigger type, tube voltage and heart rate on calcified plaque imaging in dual source cardiac computed tomography: phantom study. BMC Medical Imaging 2014 14:30

\section{Submit your next manuscript to BioMed Central and take full advantage of:}

- Convenient online submission

- Thorough peer review

- No space constraints or color figure charges

- Immediate publication on acceptance

- Inclusion in PubMed, CAS, Scopus and Google Scholar

- Research which is freely available for redistribution 